\title{
CIRCADIAN RHYTHMS OF LOCOMOTOR ACTIVITY IN THE NILE TILAPIA OREOCHROMIS NILOTICUS
}

Luisa María Vera ${ }^{\mathrm{a}}$, Louise Cairns ${ }^{\mathrm{a}}$, Francisco Javier Sánchez-Vázquez ${ }^{\mathrm{b}}$, Hervé $\operatorname{Migaud}^{\mathrm{a} *}$

${ }^{a}$ Reproduction and Genetics Group, Institute of Aquaculture, University of Stirling. Stirling, UK.

${ }^{\mathrm{b}}$ Department of Physiology, Faculty of Biology, University of Murcia, 30100 Murcia, Spain.

Running title: Circadian rhythms in tilapia

*Corresponding author:

Dr. Hervé Migaud

Institute of Aquaculture, University of Stirling

FK9 4LA, Stirling, UK

Tel. 00441786467886

Fax. 00441786472133

E-mail: herve.migaud@stir.ac.uk 


\section{ABSTRACT}

The Nile tilapia behavioural rhythms were investigated to better characterize its circadian system. To do so, the locomotor activity patterns of both male and female tilapia reared under a 12: 12-h light-dark (LD) cycle were studied, as well as the existence of endogenous rhythmicity under free-running conditions (DD and 45-min LD pulses) in males. When exposed to an LD cycle, the daily pattern of activity differed between individuals: some fish were diurnal, some nocturnal and a few displayed an arrhythmic pattern. This variability would be typical of the plastic circadian system of fish and reproductive events clearly affected the behavioural rhythms of female tilapia, a mouthbrooder teleost species. Under DD, 50\% (6 out of 12) of male fish showed circadian rhythms with an average period (tau) of $24.1 \pm 0.2 \mathrm{~h}$ whereas under the 45min LD pulses 58\% (7 out of 12) of fish exhibited free-running activity rhythms and tau was $23.9 \pm 0.5 \mathrm{~h}$. However, interestingly in this case activity was always confined to the dark phase. Furthermore, when the LD cycle was reversed a third of the experimental fish showed gradual resynchronization to the new phase, taking 7-10 days to be completely re-entrained. Taken altogether these results suggest the existence of an endogenous circadian oscillator that controls the expression of locomotor activity rhythms in the Nile tilapia, although its anatomical localization remains unknown.

Keywords: Circadian rhythms, locomotor activity, Nile tilapia, photoperiod. 


\section{INTRODUCTION}

The geophysical cycles created by the movements of Earth are responsible for the recurrent changes in daily light-dark (LD) cycles experienced by living organisms. Under such cycles the animals have developed behavioural and physiological mechanisms to anticipate predictable changes, optimizing biological processes (Daan, 1981; Godman, 2001; Kumar, 1997). Environmental changes act as synchronizers, which,entrain biological rhythms in their periodicity, amplitude and phase (Aschoff, 1981; Rensing \& Ruoff, 2002). As previously suggested, several conditions have to be met for an environmental factor to be considered as a real synchronizer (zeitgeber): once the animal is exposed to the external factor there must be a stable phase relationship between the rhythm and the zeitgeber and once the latter is suppressed the biological rhythm should free-run from the phase previously determined by the zeitgeber (Moore-Ede et al., 1982). In vertebrates, biological rhythms have been classified according to their periodicity (ultradian, circadian, infradian). However, to date work has mainly focused on rhythms related to daily environmental changes (i.e. circadian rhythms).

Under an LD cycle fish show daily patterns of locomotor activity that can be classified into several types: diurnal, nocturnal, crepuscular and combination of them (Eriksson, 1978; Herrero et al., 2003; Schulz and Leuchtenberger, 2006). However, in some teleosts species the characterisation of the daily pattern of activity is not straightforward. Thus, different individuals within the same species can show a great variability in their daily activity patterns (Helfman, 1993).

To investigate the existence of endogenous control of the behavioural rhythms in fish, constant environmental conditions are usually used (e.g. constant darkness, DD or constant illumination LL) or ultradian pulses (very short light-dark photocycles) 
(Eriksson and van Veen, 1980; Sánchez-Vázquez et al., 1995a; Sánchez-Vázquez et al., 1996; Reebs, 2002). Animals exposed to such conditions lose external entrainment and their circadian rhythms can free-run, with an approximate period (tau) of 24 hours in the case of circadian rhythms (Aschoff, 1981; Edmunds, 1988). Such rhythms are selfsustained during at least some days after the animal is separated from its external zeitgeber, thus indicating its endogenous control (Aschoff, 1960; Iigo and Tabata, 1996; Sánchez-Vázquez et al., 1995a). In mammals these circadian rhythms can be sustained under constant conditions for up to a month, whereas in teleost fish they usually disappear after a few days (Iigo and Tabata, 1996; Naruse and Oishi, 1994; Nishi, 1990). The demonstration of endogenous timing mechanisms can also be tested by phase-shifting the zeitgeber and monitor the display of transient cycles of activity (Reebs, 2002).

The Nile tilapia (Oreochromis niloticus) is a tropical freshwater species that inhabits a variety of shallow freshwater environments and is one of the most commercially important species for the world wide aquaculture industry (El-Sayed \& Kawanna, 2007). Previous investigations have proposed that this species could be endowed with a circadian system in which the pineal gland would not be light sensitive and might lack an independent circadian pacemaker, as the daily melatonin rhythm was abolished after bilateral ophtalmectomy (Migaud et al., 2007). However, the regulatory mechanism of behavioural daily rhythms in tilapia has not been yet investigated.

Due to the reproductive strategy of Nile tilapia (mouth-breeding), this study mainly focused on male fish to avoid spawning to interfere with activity rhythms. The objectives of this work were 1) to characterize the daily locomotor rhythms of male tilapia reared under an LD cycle, 2) to elucidate the existence of endogenous control of male tilapia activity rhythms by investigating circadian rhythmicity under constant 
photic conditions (DD) or ultradian pulses and studying resynchronization after phaseshifting the LD cycle and 3) to confirm the influence of spawning on the daily locomotor rhythms in female tilapia.

\section{MATERIALS AND METHODS}

\subsection{Animals and housing}

The present study was carried out in the tropical fresh-water aquarium of the Institute of Aquaculture at the University of Stirling (UK). A total of 12 males and 11 females Nile tilapia from the same stock (15 months old, $150 \mathrm{~g}$ mean body weight) red strain bred in the facilities were housed individually in rectangular $114 \mathrm{~L}$ glass tanks (54cm x 46cm x 46cm) within light proof compartments. Each tank was provided with a constant flow of well aerated freshwater and the temperature of the water was kept constant during the experiment at $27^{\circ} \mathrm{C}$.

Animals were acclimatized to a 12:12 h LD cycle for two weeks before the start of the experiments. Lighting was provided by a $60 \mathrm{~W}$ pearl bulb (Lampways Triple Plus, UK) placed above tanks. Light intensity during the photophase was $0.6 \mathrm{~W} \cdot \mathrm{cm}^{-2}$ (equivalent to approximately 100 lux) and photoperiod was controlled by a timer.

All experiments were carried out in accordance with the Animal (Scientific Procedures) Act 1986 UK under the approval of the local ethical review board and in accordance with the international ethical standards of the journal (Portaluppi et al., 2008).

\subsection{Activity monitoring}

To record the locomotor activity each aquarium was equipped with one infrared photocell (E3Z-D67, OMRON, China) placed in the centre of the front glass wall. Previously, the position of the photocell had been optimised. Photocells were connected to a motherboard (USB-1024HLS, Measurement Computing, USA) plugged to a 
computer. Every time a fish interrupted the infrared light beam it produced an output signal that was recorded and stored in 10 minute bins using a specialized software (DIO98USB, University of Murcia, Spain).

\subsection{Experimental design}

In a first experiment 12 male tilapia were reared under a 12:12 h LD cycle for 2 weeks to characterize their daily activity rhythms before exposing them to DD conditions for 3 weeks in order to study circadian rhythmicity. Thereafter, fish were resynchronized to the $\mathrm{LD}$ cycle for 2 weeks and exposed to an ultradian LD cycle (45:45 min LD) for 2 additional weeks. Subsequently, the 12:12 h LD was restored again for 2 weeks and finally reversed (lights on at 20:00 h and off at 08:00 h) to characterize the resynchronization to the new photocycle phase.

In a second experimental phase 11 female tilapia were exposed to a 12:12 h LD cycle for 3 weeks, with lights on at 08:00 h and off at 20:00 h. Throughout the experiment spawning events were recorded to determine their potential effects on locomotor activity.

In both experiments, fish were fed at random times during the photophase every 2 days to avoid feeding from acting as a potential synchronizer.

\subsection{Data analysis}

Locomotor activity records were stored in a computer and analyzed using a software package for chronobiological studies (El Temps ${ }^{\circledR}$, Prof. A. Díez-Noguera, University of Barcelona). In order to define whether individual fish were diurnal, nocturnal or arrythmic during the LD phase, statistical differences between mean diurnal and nocturnal activity were analyzed by t-Student test using Microsoft Excel (statistically significant threshold of $\mathrm{P}<0.05$ ). When no statistical difference between diurnal and nocturnal activities was observed for a given fish, it was classified as 
arrhythmic. When a statistical difference was found the fish was either defined as diurnal or nocturnal depending on when locomotor activity occurred the most (day or night). The period length (tau) of free-running rhythms was determined by SokoloveBushell periodogram analysis at a confidence level of 95\%, for which El Temps was also used.

\section{RESULTS}

\subsection{Daily and circadian rhythms of locomotor activity in male tilapia}

Male tilapia showed inter-individual differences when kept under an LD cycle (Table I): 4 out of 12 fish were mostly diurnal (Fig. 1A), with an average locomotor activity of $70.3 \pm 7.4 \%$ occurring during the photophase, 5 were mainly nocturnal (Fig. 1B), with an average activity of $64.4 \pm 4.5 \%$ displayed during the scotophase and 3 were defined as arrhythmic (Fig. 1C).

Under both DD and ultradian pulses the appearance of free-running rhythms differed between fish, some of them showed self-sustained rhythms once the external 12:12 h LD cycle was removed whereas others were arrhythmic during the first few days and then started to show endogenous activity rhythms.

Under DD, 6 out of 12 male tilapia exhibited self-sustained circadian rhythms with locomotor activity free-running with an average tau of $24.1 \pm 0.2 \mathrm{~h}$, ranging from 23.5 to 25.2 h (Table II). Figure 2 shows two representative fish with locomotor freerunning rhythms longer than $24 \mathrm{~h}$. There was no prevalence of fish with diurnal or nocturnal activity under an LD cycle displaying rhythmicity during the DD phase (2 were diurnal, 3 nocturnal and 1 arrythmic).

When fish were exposed to 45 -min LD pulses, 7 out of 12 male tilapia showed circadian rhythmicity. Locomotor activity free ran with an average tau of $23.9 \pm 0.5 \mathrm{~h}$, 
ranging from 21.0 to $24.7 \mathrm{~h}$ (Table II). Locomotor activity from two experimental fish is presented in Fig. 3. The activity rhythms of the first fish started to free-run 6 days after the LD cycle was removed and showed circadian rhythms with tau shorter than $24 \mathrm{~h}$ (Fig. 3A). On the contrary, locomotor activity of the second fish free-ran once the fish was exposed to the 45-min LD pulses and tau was longer than $24 \mathrm{~h}$ (Fig. 3B). Underlying the free-running rhythms, when ultradian pulses were applied, locomotor activity of tilapia was mostly confined to the dark phase (Fig.4). Interestingly, this was observed in both diurnal and nocturnal tilapia, when reared under a 12:12 h LD cycle.

Finally, the daily LD cycle was reversed in the last experimental phase to investigate how fish resynchronized after the zeitgeber phase-shifting. Three out of 12 fish changed immediately the phase of their locomotor activity rhythm, so that it coincided with the new phase of the photocycle (Fig. 5A). However, 4 experimental tilapia showed a gradual resynchronization to the new phase, as transient cycles of activity were observed. The day following the shift from LD to DL, fish were active at the same time as before the phase-shifting but day after day they started to be active earlier and slowly resynchronized to the new phase (Fig. 5B). Other tilapia did not resynchronize at all, e.g. one diurnal individual became arrhythmic after the LD cycle was reversed but after a few days was active during the same phase as before the shifting, although then it corresponded to the scotophase (Fig. 5C).

\subsection{Effect of spawning on the daily rhythms of locomotor activity in female Nile tilapia}

When female tilapia were reared under an LD cycle, the daily distribution of activity showed high variability among individuals: 5 out of 11 fish displayed a mostly diurnal locomotor activity pattern (with $74.4 \pm 6.3 \%$ of their activity occurring during the photophase), 1 tilapia showed nocturnal rhythmicity (68.0\% of activity displayed at 
night) and 5 fish were arrhythmic throughout the experiment (data not shown). Ten out of eleven individuals spawned during the experiment, four of them twice. The reproductive activity clearly affected the daily locomotor rhythm in nine of the experimental fish. Among the four individuals which displayed activity rhythms (either diurnal or nocturnal), two showed a reduction in activity from the egg incubation stage until few days later (Fig. 6A), one reduced its activity prior spawning and then became arrhythmic (Fig. 6B) and the last one showed both a reduction in activity and arrhythmicity from 3-4 days prior spawning and during egg incubation. All remaining individuals (5), which were initially arrhythmic, remained so and showed a reduction of activity during and several days following egg incubation.

\section{DISCUSSION}

This study provides interesting preliminary results on locomotor activity rhythms in the Nile tilapia (O. niloticus).

Male and female tilapia showed large inter-individual variations of daily activity distribution when reared under an LD cycle. From a total of 23 fish used in our experiments, 9 were mostly diurnal, 6 nocturnal and 8 were arrhythmic. In males, 9 out of 12 fish exhibited daily rhythms of locomotor activity (45\% of which were diurnal) whereas in females only 6 out of 11 fish showed rhythmicity in their activity (83\% were diurnal). The present results are in contrast to the general knowledge that has defined the Nile tilapia as a diurnal species and differences between males and females could either be sex-dependent or due to inter-individual variations.

The variability of behavioural patterns observed in the present study is in accordance with previously published data obtained in other fish species. Indeed, when goldfish (Carassius auratus) were exposed to an LD 12:12 h cycle, the daily rhythm of 
locomotor activity also differed between individual fish: some were diurnal and others were nocturnal (Sánchez-Vázquez et al., 1996). Furthermore, sea bass (Dicentrarchus labrax) showing diurnal feeding rhythms could be turned into nocturnal by restricting food availability to the night period (Sánchez-Vázquez et al., 1995a). In sharpsnout seabream (Diplodus puntazzo) spontaneous shifts from diurnal to nocturnal patterns of locomotor activity and vice versa were reported, as well as the existence of phase independence between locomotor and feeding rhythms (Vera et al., 2006). In contrast, other teleost species show clear daily rhythms of locomotor activity, such as the zebrafish and the tropical fish Halichoeres chrysus which mostly confine their activity to the photophase under an LD cycle (Gerkema et al., 2000; Hurd et al., 1998) whereas the tench (Tinca tinca) shows a strictly nocturnal pattern, even under extremely short photoperiods (e.g. LD 22: 2 h) (Herrero et al., 2003). Therefore, it has been generally accepted that activity patterns in fish show a strong plasticity (Ali, 1992; Madrid et al., 2001; Reebs, 2002). Freshwater teleosts have been proposed to have a more flexible circadian system than marine fish, probably due to the relative instability of their environment (Reebs, 2002). Indeed, in the current study, large inter-individual variability of the tilipia behavioural patterns was observed. This could suggest that the circadian clocks in tilapia might not be as robust as seen in other vertebrate species, since they do not always maintain activity to a particular phase of the LD cycle (Gallistel, 1990; Reebs, 2002). Interestingly, the fact that some fish showed nocturnal locomotor activity rhythms raises the question whether these individuals would feed during the night if they were given the chance. The study of daily feeding rhythms in this species would be certainly an interesting subject to pursue.

Circadian rhythmicity in locomotor activity of tilapia reared under DD and ultradian light pulses was observed in our study, suggesting the existence of a circadian 
clock in this species that regulates its activity. Under DD, 50\% of the fish exhibited self-sustained activity rhythms and 58\% under a 45:45 min LD cycle. In tench $41 \%$ of experimental fish showed circadian rhythmicity under DD (Herrero et al., 2003) and in sharpsnout sea bream only a third of the individuals did (Vera et al., 2006). In mammals, however, the ratio of animals showing circadian rhythmicity under constant conditions is usually higher and the self-sustained rhythms can persist longer (Benstaali et al., 2001). Some tilapia showed free-running rhythms immediately after the removal of the LD 12:12 h cycle whereas others were arrhythmic during the first few days under constant photic conditions and then showed free running locomotor activity. This was also observed in zebrafish when transferred from a daily LD cycle to DD (Hurd et al., 1998). The period length also differed among individuals and although some fish showed circadian rhythms both under DD and 45:45 min LD others only did under one of the two experimental conditions. Similar results were obtained when goldfish and zebrafish circadian activity rhythms were studied (Iigo and Tabata, 1996; Hurd et al., 1998), supporting that the circadian system of teleosts could be considered as a multiphotoreceptor and multioscillator system and that the coupling between the different oscillators may differ between individuals within the same species depending on the physiological and environmental conditions (Iigo et al., 1994; Kavaliers, 1980; Kavaliers, 1981; Pittendrigh, 1974; Tabata, 1992; Underwood, 1994).

Interestingly, when tilapia were exposed to ultradian light pulses we observed that underlying to the expression of circadian rhythms the locomotor activity was confined to the dark phase of the 45:45 min LD cycle, in both fish displaying a diurnal and nocturnal pattern during the 12:12 h LD cycle. This could be due to the splitting of the circadian rhythm into two components, so that the unimodal pattern would become bimodal (Earnest, 1982; Pittendrigh, 1974). However, the existence of negative light 
masking could also explain this phenomenon (Mrosovsky, 1999). Masking involves the passive influence of the environmental factors on the behavioural patterns without affecting the endogenous oscillators (Aschoff, 1981) and its effect was observed in goldfish locomotor and feeding rhythms when exposed to ultradian pulses (SánchezVázquez et al., 1996). Another way to investigate the existence of a circadian clock is to phase-shift the zeitgeber. In the present study when the LD cycle was suddenly reversed to DL a third of the experimental fish showed a gradual re-entrainment to the new phase, taking 7-10 days to resynchronize completely. This has also been reported in the hagfish Eptatretus burger (Ooka-Souda et al., 1985) and golden shiners Notemigonus crysoleucas (Laguë and Reebs, 2000) although most of fish species studied to date usually displayed only one day of transient activity (Godin, 1981; Nelson and Jhonson, 1970; Sánchez-Vázquez et al., 1995a; Tabata et al., 1989).

Previous studies have reported that tilapia might lack an independent circadian pacemaker as after bilateral ophtalmectomy the day-night melatonin rhythm was suppressed (Migaud et al., 2007) although when fish were exposed to DD, a strong circadian melatonin rhythm was maintained for 18 days (Martínez-Chávez et al., 2008), suggesting the existence of endogenous oscillators in this species. Our study supported this hypothesis as endogenous activity rhythms were observed under constant conditions (DD) and ultradian pulses, as well as the existence of gradual resynchronization after phase-shifting the LD cycle.

Finally, a clear effect of spawning was observed on the daily rhythms of activity in female tilapia, characterised by an important reduction of activity before, during and/or following spawning and causing arrhythmicity in some cases. Tilapia is a mouthbrooder species able to spawn multiple times throughout the year. When females do not have males to fertilise their eggs they hold the unfertilised eggs in their mouth 
for 1-2 days and then spit them out (El-Sayed, 2006). Previous investigations in cardinalfish (Apogon fragilis and Apogon leptachanthus) suggested that mouthbrooding can increase the rate of oxygen consumption, reduced the ability of fish to take up oxygen and impaired the capacity for sustained aerobic swimming (Östlund-Nilsson and Nilsson, 2004). These results could explain the change of activity patterns observed in female tilapia from the present study. The fact that reproductive events also caused arrhythmicity in some tilapia a few days before and after mouthbrooding agrees with previous observations in other species that during the spawning season activity is displayed throughout the day (Helfman, 1981).

To conclude, the present study suggests that tilapia is endowed with a flexible circadian system, which endogenously controls the expression of behavioural rhythmic patterns, although further investigations will be needed to elucidate the mechanisms at work.

\section{ACKNOWLEDGEMENTS}

The authors would like to thank the staff of the University of Stirling Tropical Aquarium for providing the fish and helping with the daily management and Dr Andrew Davie for his constructive comments to improve the present manuscript. Financial support was provided by the Institute of Aquaculture at the University of Stirling as well as by “Fundación Séneca” and “Agencia Regional de Ciencia y Tecnología” for the postdoctoral grant awarded to L.M. Vera.

Declaration of interest: The authors report no conflicts of interest. The authors alone are responsible for the content and writing of the paper. 


\section{REFERENCES}

Ali, MA. (1992). Rhythms in fishes. New York: Plenum Press.

Aschoff, J. (1960). Exogenous and endogenous components in circadian rhythms. Cold Spring Harbor Symp. Quant. Biol. 25: 11-28.

Aschoff, J. (1981). Biological rhythms. In Aschoff, J. (ed). Handbook of Behavioral Neurobiology, vol 4: Biological Rhythms. New York: Plenum Press, 563 pp.

Benstaali C, Mailloux A, Bogdan A, Auzéby A, Touitou, Y. (2001). Circadian rhythms of body temperature and motor activity in rodents. Their relationships with the lightdark cycle. Life Sci. 68: 2645-2656.

Daan S. (1981). Adaptative daily strategies in behaviour. In Aschoff J. (ed). Handbook of behavioural neurobiology, vol 4, biological rhythms. New York: Plenum Press, pp. 275-298.

Earnest DJ, Turek FW. (1982). Splitting of the circadian rhythm of activity in hamsters : effects of exposure to constant darkness and subsequent re-exposure to constant light. $J$. Comp. Physiol. 145: 405-411.

Edmunds LN. (1988). Cellular and molecular basis of biological clocks: models and mechanisms for biological timekeeing. Berlin: Springer Verlag.

El-Sayed AM. (2006). Tilapia Culture. Oxfordshire: CABI Publishing.

El-Sayed AM, Kawanna M. (2007). Effects of photoperiod on growth and spawning efficiency of Nile Tilapia (Oreochromis niloticus L.) broodstock in a recycling system. Aquac. Res. 38: 1242-1247.

Eriksson LO. (1978). Nocturnalism vs diurnalism - dualism within fish individuals. Thorpe JE (ed). Rhythmic activity of fishes. New York: Academic Press, pp. 69-89. 
Eriksson LO, van Veen T. (1980). Circadian rhythms in the brown bullhead, Ictalurus nebulosis (Teleostei): evidence for an endogenous rhythm in feeding, locomotor and reaction time behaviour. Can. J. Zool. 58: 1899-1907.

Gallistel CR. (1990). The organization of learning. Cambridge, MA: MIT Press.

Gerkema MP, Videler JJ, de Wiljes J, van Lavieren H, Gerritsen H, Karen M. (2000). Photic entrainment of circadian activity patterns in the tropical labrid fish Halichoeres chrysus. Chronobiol. Int. 17: 613-622.

Godin JGJ. (1981). Circadian rhythms of swimming activity in the juvenile pink salmon (Onchorhynchus gorbuscha). Mar. Biol. 64: 341-349.

Godman BD. (2001). Mammalian photoperiodic system: formal properties and neuroendocrine mechanisms of photoperiodic time measurement. J. Biol. Rhythms 16: 283-301.

Helfman GS. (1981). Twilight activities and temporal structure in a freshwater fish community. Can. J. Fish. Aqua. Sci. 38: 1405-1420.

Helfman, G.S. (1993). Fish behaviour by day, night and twilight. Pitcher TJ (ed). Behaviour of teleost fishes. London: Chapman and Hall, pp. 479-512.

Herrero MJ, Madrid JA, Sánchez-Vázquez FJ. (2003). Entrainment to light of circadian activity rhythms in tench (Tinca tinca). Chronobiol. Int. 20: 1001-1017.

Hurd MW, Debruyne J, Straume M, Cahill GM. (1998). Circadian rhythms of locomotor activity in zebrafish. Physiol. Behav. 65: 465-472.

Iigo M, Tabata M. (1996). Circadian rhythms of locomotor activity in the goldfish Carassius auratus. Physiol. Behav. 60: 775-781.

Iigo M, Kezuka H, Suzuki T, Tabata M, Aida K. (1994). Melatonin signal transduction in the goldfish, Carassius auratus. Neurosci. Biobehav. Rev. 18: 563-569. 
Kavaliers M. (1980). Circadian locomotor activity rhythms of the brubot, Lota lota: seasonal differences in period length and the effect of pinealectomy. J. Comp. Physiol. A 136: 215-218.

Kavaliers M. (1981). Circadian organization in the white sucker Catostomus commersoni: the role of the pineal organ. Comp. Biochem. Physiol. 68A: 127-129.

Kumar V. (1997). Photoperiodism in higher vertebrates: an adaptive strategy in temporal environment. Indian J. Exp. Biol. 35: 427-437.

Laguë M, Reebs SG. (2000). Phase-shifting the light-dark cycle influences foodanticipatory activity in golden shiners. Physiol. Behav. 70: 55-59.

Madrid JA, Boujard T, Sánchez-Vázquez FJ. (2001). Feeding rhythms. Houlihan DF, Boujard T, Jobling M. (eds). Food intake in fish. Oxford: Blackwell Science, pp. 189215.

Martínez-Chávez CC, Al-Khamees S, Campos-Mendoza A, Penman DJ, Migaud H. (2008). Clock-controlled endogenous melatonin rhythms in Nile tilapia (Oreochromis niloticus niloticus) and African catfish (Clarias gariepinus). Chronobiol. Int. 25: 31-49. Migaud H, Davie A, Martínez-Chávez CC, Al-Khamees S. (2007). Evidence for different photic regulation of pineal melatonin synthesis in teleosts. J. Pineal Res. 43: 327-335.

Moore-Ede MC, Sulzman FM, Fuller CA. (1982). The clocks that time us. Cambridge: Harvard University Press.

Mrosovsky N. (1999). Masking: history, definitions, and measurement. Chronobiol. Int. 16: $313-354$

Naruse M, Oishi T. (1994). Effects of light and food as zeitgebers on locomotor activity rhythms in the loach, Misgurnus anguillicaudatus. Zool. Sci. 11: 113-119. 
Nelson DR, Jhonson RH. (1970). Diel activity rhythms in the nocturnal, bottomdwelling sharks Heterodontus francisci and Cephaloscyllium ventriosum. Copeia 732739.

Nishi G. (1990). Locomotor activity rhythm in four wrasse species under varying light conditions. Jpn. J. Ichtyol. 37: 170-181.

Ooka-Souda S, Kabasawa H, Kinoshita S. (1985). Circadian rhythms in locomotor activity in the hagfish, Eptatretus burger, and the effect of reversal of light-dark cycle. Zool. Sci. 2: 749-754.

Östlund-Nilsson S, Nilsson GE. (2004). Breathing with a mouth full of eggs: respiratory consequences of mouthbrooding in cardinalfish. Proc. R. Soc. Lond. B 271: 1015-1022. Pittendrigh CS. (1974). Circadian oscillations in cells and the circadian organization of multicellular systems. Schmitt FO, Worden FG (eds). The neurosciences: third study program. Cambridge, MA: MIT Press, pp. 437-458.

Portaluppi F, Touitou Y, Smolensky M. (2008). Ethical and methodological standards for laboratory and medical biological rhythm research. Chronobiol. Int. 25: 999-1016. Reebs SG. (2002). Plasticity of diel and circadian activity rhythms in fishes. Rev. Fish Biol. Fisher. 12: 349-371.

Rensing L, Ruoff P. (2002). Temperature effect on entrainment, phase shifting and amplitude of circadian clocks and its molecular bases. Chronobiol. Int. 19: 807-864. Sánchez-Vázquez FJ, Madrid JA, Zamora S. (1995a). Circadian rhythms of feeding activity in sea bass, Dicentrarchus labrax L.: dual phasing capacity of diel demandfeeding pattern. J. Biol. Rhythms 10: 256-266.

Sánchez-Vázquez FJ, Zamora S, Madrid JA. (1995b). Light-dark and food restriction cycles in sea bass: effect of conflicting zeitgebers on demand-feeding rhtyhms. Physiol. Behav. 58: 705-714. 
Sánchez-Vázquez FJ, Madrid JA, Zamora S, Iigo M, Tabata M. (1996). Demand feeding and locomotor circadian rhythms in the goldfish, Carassius auratus: dual and independent phasing. Physiol. Behav. 60: 665-674.

Schulz UH, Leuchtenberger C. (2006). Activity patterns of South American silver catfish (Rhamdia quelen). Braz. J. Biol. 66: 565-574.

Tabata M. (1992). Photoreceptor organs and circadian locomotor activity in fishes. Ali MA (ed). Rhythms in fishes. New York, Plenum Press, pp. 223-234.

Tabata M, Minh-Nyo M, Niwa H, Oguri M. (1989). Circadian rhythm of locomotor activity in a teleost, Silurus asotus. Zool. Sci. 6: 367-375.

Underwood H. (1994). The pineal and circadian organization in fish, amphibians and reptiles. Reiter RJ (ed). The pineal gland, vol. III: Extra-reproductive effects. CRC Press, Boca Raton, FL, pp. 1-25.

Vera LM, Madrid JA, Sánchez-Vázquez FJ. (2006). Locomotor, feeding and melatonin rhythms in sharpsnout seabream (Diplodus puntazzo). Physiol. Behav. 88: 167-172. 
Table I: Percentage of activity displayed during the photophase by male tilapia under a 12:12 h LD cycle and ultradian pulses. D: diurnal; N: nocturnal; A: arrhythmic.

\begin{tabular}{|c|c|c|}
\hline Fish & $\begin{array}{c}\text { \% Activity during photophase } \\
(12: 12 \mathrm{~h} \mathrm{LD})\end{array}$ & $\begin{array}{c}\text { \% Activity during photophase } \\
(45: 45 \text { min LD })\end{array}$ \\
\hline 1 & $58 ; \mathrm{D}$ & 26 \\
\hline 2 & $83 ; \mathrm{D}$ & 21 \\
\hline 3 & $21 ; \mathrm{N}$ & 18 \\
\hline 4 & $45 ; \mathrm{A}$ & 19 \\
\hline 5 & $57 ; \mathrm{D}$ & 42 \\
\hline 6 & $42 ; \mathrm{N}$ & 23 \\
\hline 7 & $43 ; \mathrm{N}$ & 48 \\
\hline 8 & $42 ; \mathrm{N}$ & 22 \\
\hline 9 & $63 ; \mathrm{A}$ & 36 \\
\hline 10 & $48 ; \mathrm{A}$ & 22 \\
\hline 11 & $28 ; \mathrm{N}$ & 18 \\
\hline 12 & $83 ; \mathrm{D}$ & 18 \\
\hline
\end{tabular}


Table II: Free-runnig rhythms period (Tau) in male tilapia under DD and ultradian pulses. NS: non significative.

\begin{tabular}{|c|c|c|}
\hline Fish & Tau under DD (h) & Tau under ultradian pulses (h) \\
\hline 1 & NS & 24.4 \\
\hline 2 & 23.8 & NS \\
\hline 3 & 24.8 & 25.2 \\
\hline 4 & 24.5 & 21.0 \\
\hline 5 & 24.2 & 24.5 \\
\hline 6 & NS & 24.7 \\
\hline 7 & 23.8 & NS \\
\hline 8 & NS & NS \\
\hline 9 & NS & NS \\
\hline 10 & NS & NS \\
\hline 11 & 23.5 & 23.5 \\
\hline 12 & NS & 24.2 \\
\hline
\end{tabular}




\section{FIGURE LEGENDS:}

Figure 1: Average diel profile of locomotor activity from three male tilapia reared under a 12: 12 h LD cycle, showing a diurnal (A), nocturnal (B) and arrhythmic (C) pattern. The white and black bars at the top of each graph indicate the light and dark periods, respectively. Data represent the mean (continuous line) + S.E.M. (dotted line) of one fish during two weeks.

Figure 2: Locomotor actograms (upper graphs) and their corresponding periodogram analysis (down) from two male tilapia subjected to DD conditions. The period of the free-running rhythm (tau) is indicated above the periodograms. Actograms are doubleplotted for better visualization.

Figure 3: Locomotor actograms (left graphs) and their corresponding periodogram analysis (right) from two male tilapia exposed to ultradian light pulses (45: 45 min LD). The period of the free-running rhythm (tau) is indicated above the periodograms.

Figure 4: Average diel profile of tilapia locomotor activity under a 45-min LD cycle. Data represent the mean (continuous line) + S.E.M. (dotted line) of twelve male tilapia during two weeks.

Figure 5: Locomotor actograms from three male tilapia exposed to different subsequent photoperiods along the experiment: 12:12 h LD, DD, 45:45 min LD and DL.

Figure 6: Locomotor actograms (left) and average diel profile activity (right) under a 12:12 h LD cycle from a nocturnal (A) and a diurnal (B) female tilapia showing spawning events during the experiment. Waveforms represent the mean (continuous lines) + S.E.M. (dotted lines) of one fish during the experiment. 
Figure 1
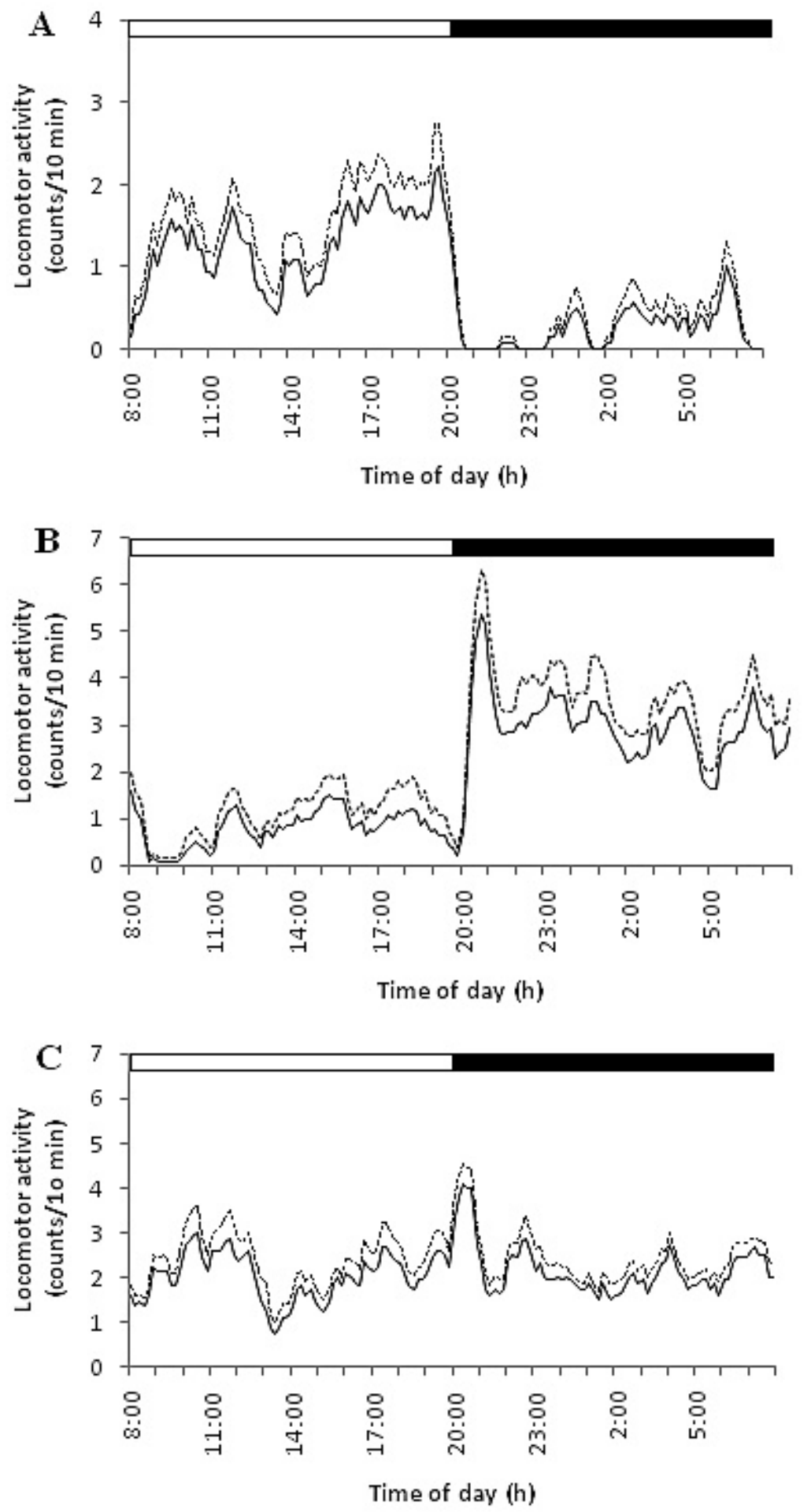
Figure 2
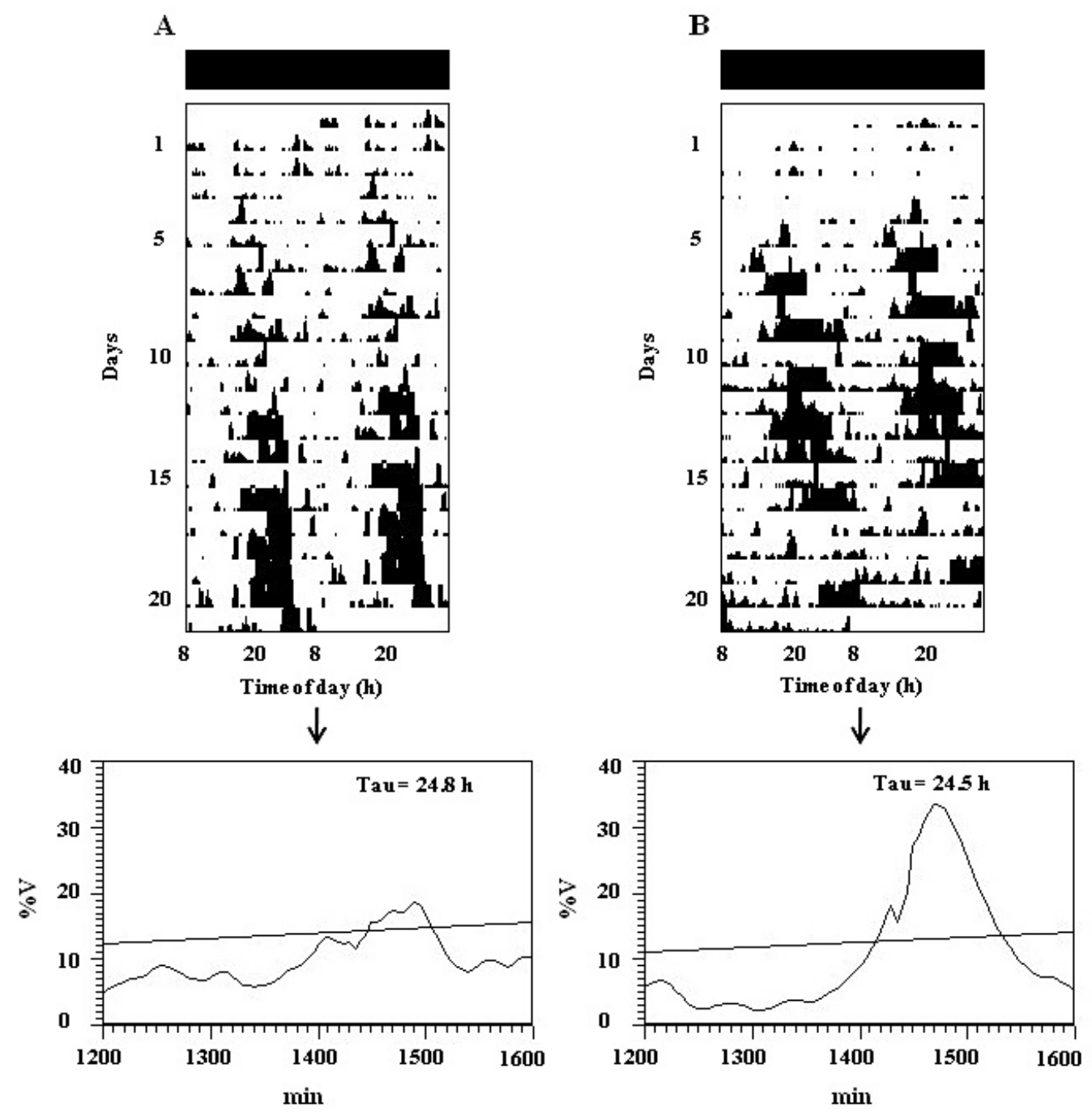
Figure 3
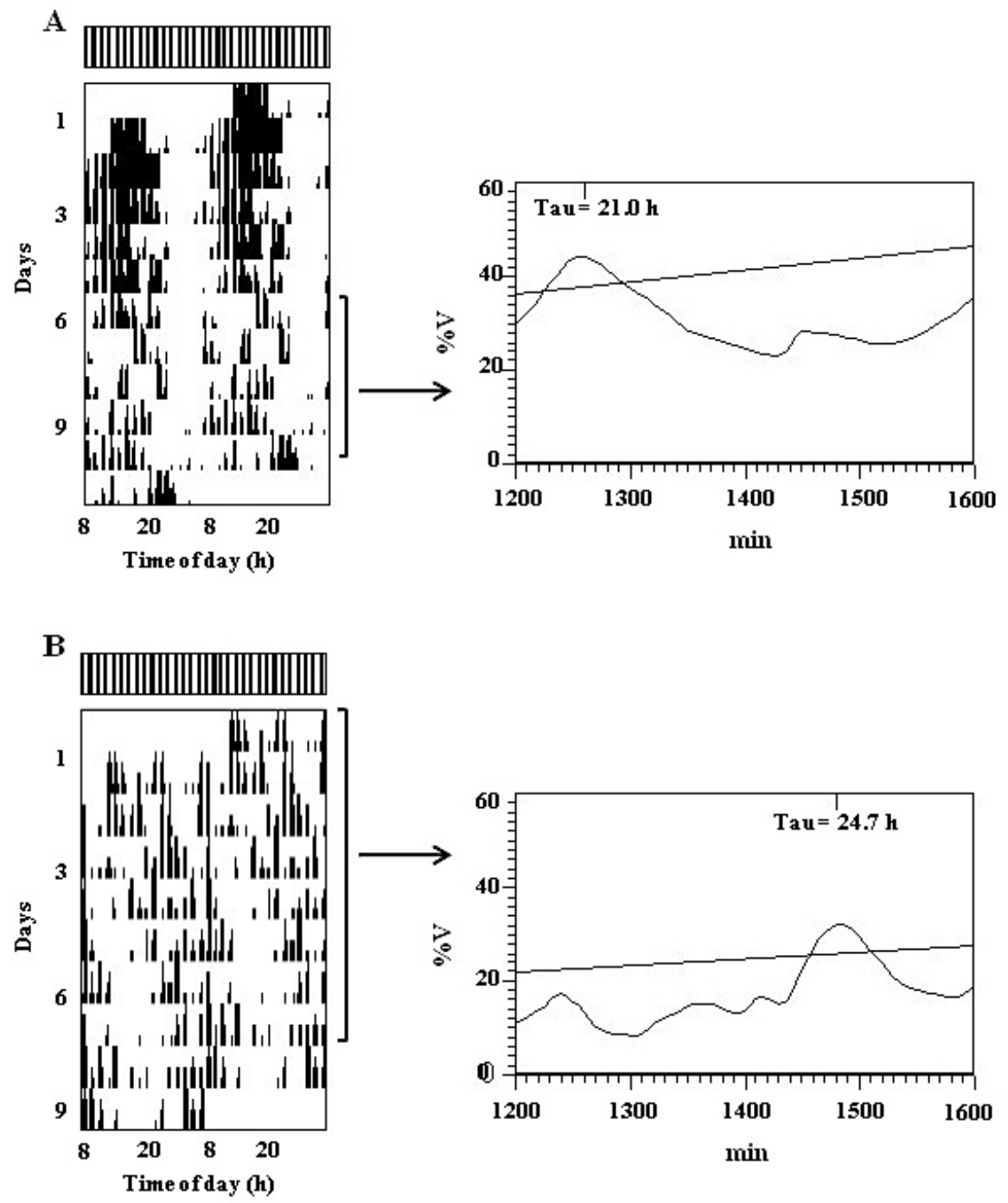
Figure 4

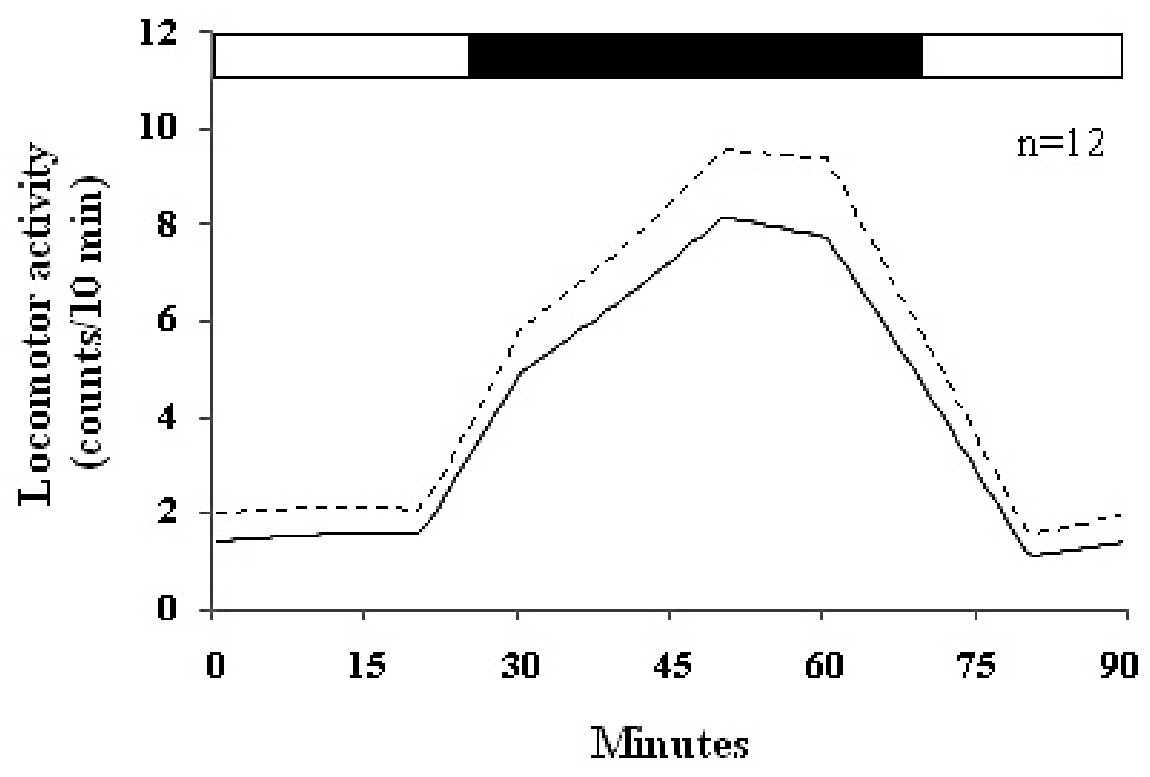


Figure 5

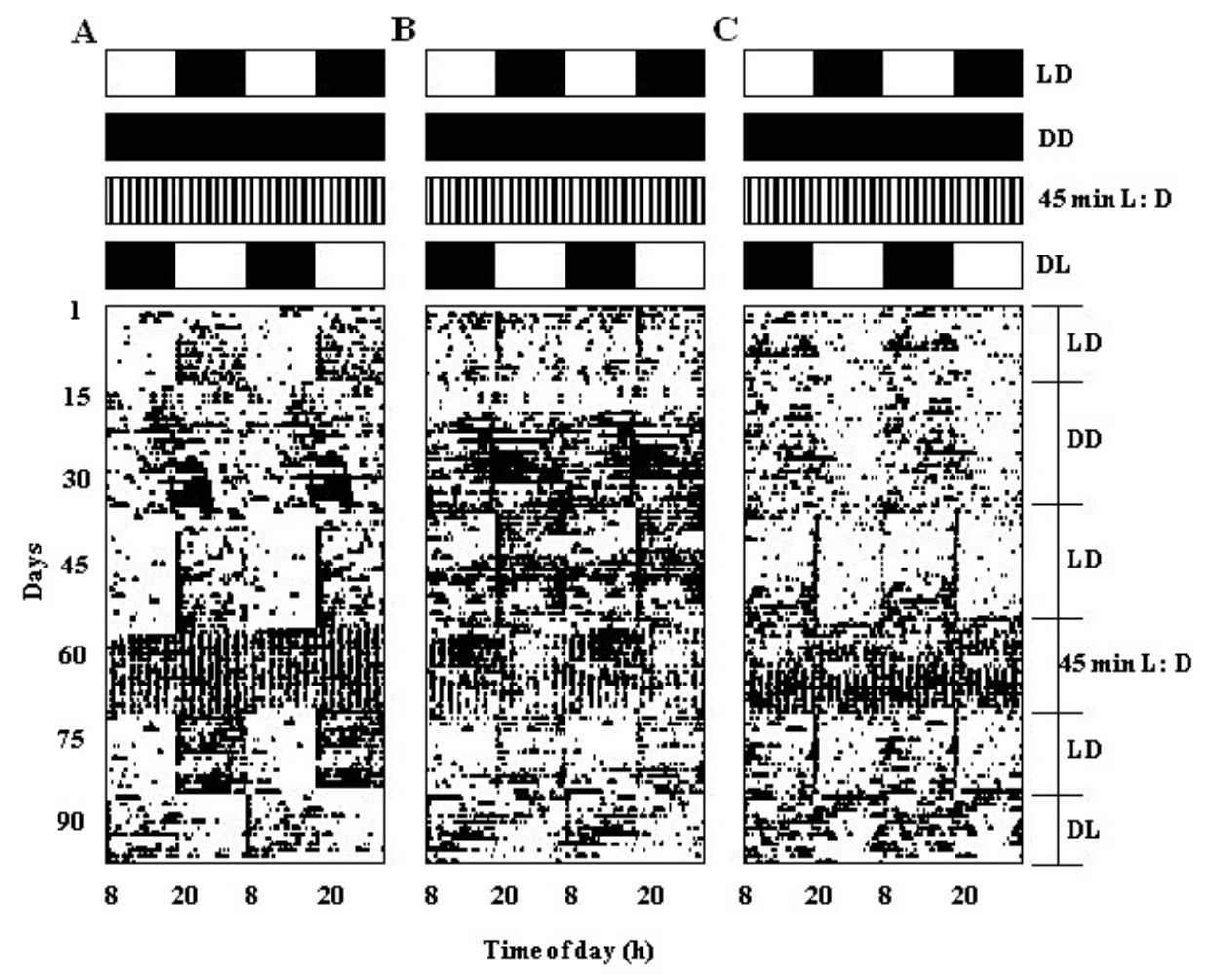


Figure 6
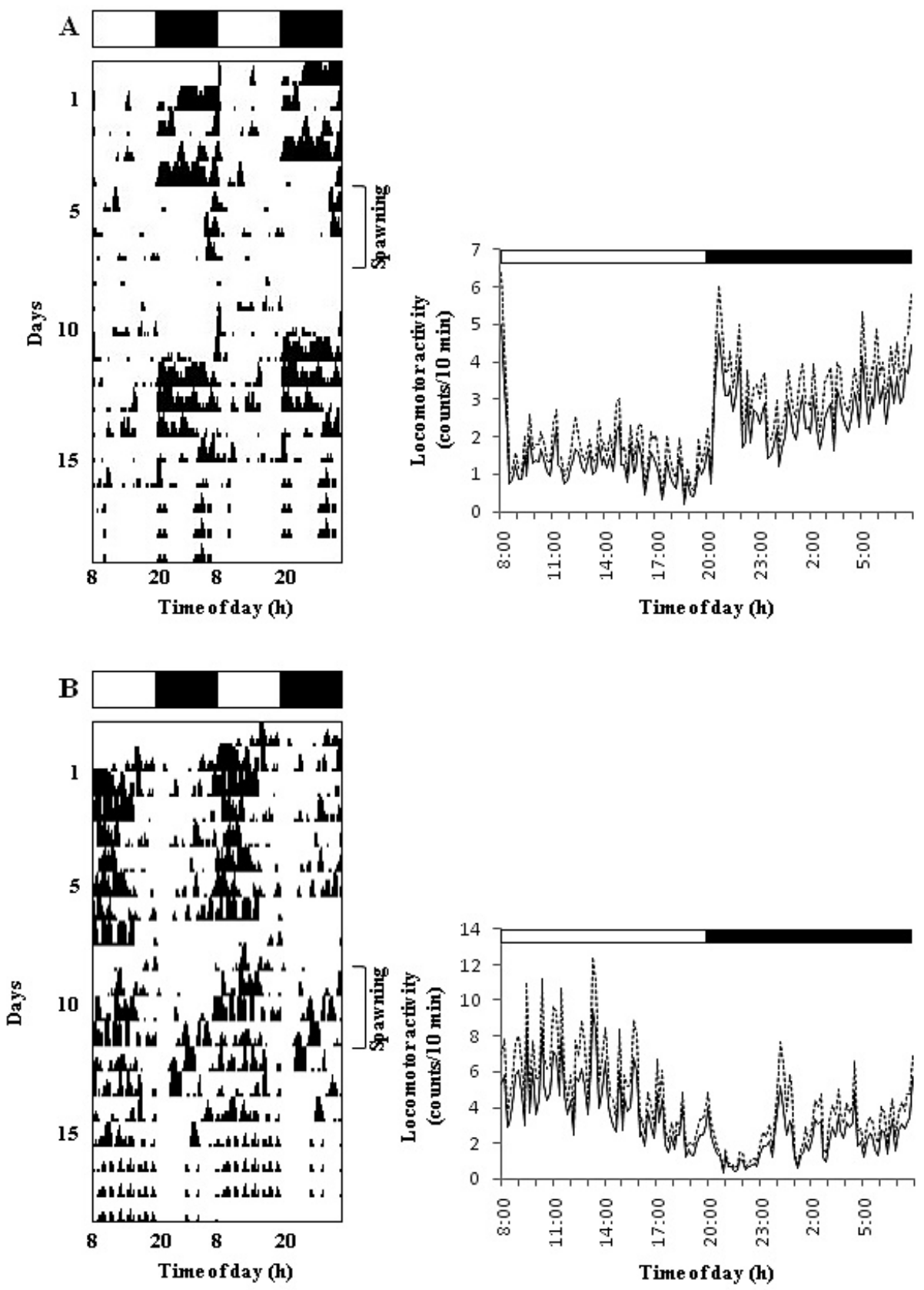